\title{
Diabetic Retinopathy Detection using Image Processing
}

\author{
R. Naveen, S. A. Sivakumar, B. Maruthi Shankar, A. Keerthana Priyaa
}

\begin{abstract}
The main objective of this method is to detect DR (Diabetic Retinopathy) eye disease using Image Processing techniques. The tool used in this method is MATLAB (R2010a) and it is widely used in image processing. This paper proposes a method for Extraction of Blood Vessels from the medical image of human eye-retinal fundus image that can be used in ophthalmology for detecting DR. This method utilizes an approach of Adaptive Histogram Equalization using CLAHE (Contrast Limited Adaptive Histogram Equalization) algorithm with open CV (Computer Vision) framework implementation. The result shows that affected $D R$ is detected in fundus image and the DR is not detected in the healthy fundus image and $98 \%$ of Accuracy can be achieved in the detection of DR.
\end{abstract}

Keywords: Adaptive Histogram Equalization, CLAHE (Contrast Limited Adaptive Histogram Equalization), DR (Diabetic Retinopathy), Image Processing.

\section{INTRODUCTION}

The processing of images by performing some operations in order to get enhanced images is called as image processing. It is widely used to diagnose the eye diseases in an easy and efficient manner. Several techniques has been developed for the early detection of DR on the basis of features such as blood vessels, hemorrhages, exudes etc., It includes the image enhancement processes like histogram equalization and adaptive histogram equalization for the detection of DR. The persistent damage caused to the retina is termed as the retinopathy. The condition of diabetic retinopathy (DR) happens with those who have diabetes that results in progressive damage to the retina. Due to high blood glucose levels it leads to the damage of small blood vessels in the retina and this may result into swelling and hemorrhage of the retina. ie., DR is a diabetes related eye disease which occurs when the blood vessels in the retina become swelled and leaks fluid which ultimately leads to vision loss. The DR is regarded as a serious sight threatening condition.

The classification of DR includes NPDR and PDR. The Non Proliferative Diabetic Retinopathy (NPDR) leads to the swelling of the blood vessels in the retina. The Proliferative Diabetic Retinopathy (PDR) leads to the formation of new blood vessels which are fragile and abnormal. The symptoms of DR includes difficulty in seeing well at night, problem in

Revised Manuscript Received on July 22, 2019.

Dr.R.Naveen, Professor and Principal, K.V.Subba Reddy College of Engineering for Women, Kurnool, AP, India

Dr.S.A.Sivakumar, Professor and Head-ECE, K.V.Subba Reddy College of Engineering for Women, Kurnool, AP, India

Dr.B.Maruthi Shankar, Associate Professor - ECE, Sri Krishna College of Engineering and Technology, Coimbatore, Tamilnadu, India

A.Keerthana Priyaa, PG Scholar, Info Institute of Engineering, Coimbatore, Tamilnadu, India seeing (or) having blurred vision, empty (or) dark spot in the center of the vision. According to the International Diabetes Foundation, the number of adults with diabetes in the world is estimated to 366 million in 2011 and in 2030 it will increase to 522 million. The number of people with type-2 diabetes is increasing to $80 \%$ in every country and India stands first with 95\% (18 million in 1995 and it will increase to 54 million in 2025). So diabetes is the major cause for DR and it may also leads to vision loss. In Southern India prevalence of DR is $12.2 \%$ to $18.03 \%$ and the people above 50 years of age affected by DR is $16.6 \%$ to $20.9 \%$. The knowledge and awareness relating to DR is very poor. Only one fifth of paramedics and one tenth of the people from the community were aware that uncontrolled diabetes is a risk factor for DR. Laser surgery called as scatter laser treatment can be done at affordable price in India to cure DR. Some of the hospitals that diagnose and treat DR are; Lotus Eye Care Hospital, Coimbatore; Apollo Hospital, Chennai \& Hyderabad; Max Super Specialty Hospital, Delhi.

\section{LITERATURE REVIEW}

\section{A. Extraction blood vessels from retinal fundus image based on fuzzy $C$-median clustering algorithm}

Thanapong, C., et al [2007] developed the extraction of blood vessels from retinal fundus image based on fuzzy $\mathrm{C}$-median clustering algorithm. This paper proposes an automated method of detection and extraction of blood vessels in retinal images [7]. The proposed algorithm is composed of three steps: Matched filtering - used to enhance visualization of the blood vessels, Fuzzy C-Median (FCMED) clustering - used to keep the spatial structure of vascular tree segments, Label filtering - used to remove misclassified pixels .This algorithm has been evaluated in terms of sensitivity and specificity, this method performs well in analyzing anatomical structures in retinal images.

\section{B. An adaptive threshold based algorithm for detection of} red lesions of diabetic retinopathy in a fundus image

Ganguly,S., et al [2014] developed the adaptive threshold based algorithm for the detection of the red lesions of diabetic retinopathy in the fundus image. This paper proposes an algorithm for detection of red lesions present in a fundus image of an eye [3]. The Red lesions include micro-aneurysms and hemorrhages which are the symptoms of DR. The proposed method describes the upper threshold and lower threshold of the red lesions for the given fundus image individually based on the local image information. Every fundus image is processed with a different 


\section{Diabetic Retinopathy Detection using Image Processing}

intensity threshold value for more accurate detection.

\section{Automatic Detection of Blood Vessel in Retinal Images}

Elbalaoui, A., et al [2016] developed the automatic detection of blood vessel in the retinal images. This paper proposes the automatic detection of the retinal blood vessels and the measurement of the vessel diameter. In which it is important for the diagnosis and the treatment of different ocular diseases including DR, glaucoma and hypertension [2].The proposed method consists of three main steps: Pre-processing of retinal images, the Vesselness filter is used to enhance the blood vessels and Finally Hessian multi-scale enhancement filter is designed from the adaptive thresholding of the output of a vesselness filter for vessels detection.

\section{D.Diabetic retinopathy detection using image-processing}

Zaman, A.U., et al [2016] developed the diabetic retinopathy detection using the image-processing. This paper proposes in detail about the diabetic retinopathy-eye disease. It also proposes a method to overcome the problem of DR [8]. By using the help of convolutional neural network (ConvNet), it is possible to detect multiple stages of severity in diabetic retinopathy.

\section{E. Automatic extraction of blood vessels and veins using laplace operator in fundus image}

Minar, J., et al [2015] developed the automatic extraction of blood vessels and veins using the laplace operator in the fundus image. This paper proposes a novel method for extraction of blood vessels [5]. By using the laplace operator and applying it on the processed image and also on the removed small segments from the image inorder to enhance the extraction of blood vessels from the fundus image. This technique analyzes the detection and evaluates the precision of the fundus images.

\section{PROPOSED METHODOLOGY}

The Adaptive Histogram Equalization (AHE) is a computer image processing technique used to improve contrast in images. It is suitable for improving the local contrast and enhancing the definitions of the edges in each region of an image. It has the tendency to over amplify noise in relatively homogenous regions of an image. A variant of the adaptive histogram equalization called as the Contrast Limited Adaptive Histogram Equalization (CLAHE) prevents this by limiting the amplification. The Contrast Limited Adaptive Histogram Equalization (CLAHE) differs from ordinary Adaptive Histogram Equalization (AHE) in its contrast limiting. In CLAHE, the contrast limiting procedure has to be applied for each neighbourhood from which a transformation function is derived. The CLAHE was developed to prevent the over amplification of noise that AHE can give rise to. This is achieved by limiting the contrast enhancement of AHE. The CLAHE method limits the amplification by clipping the histogram at a predefined value before computing the Cumulative Distribution Function (CDF).

of different wavelength. One type of the cone is mainly sensitive to the red light, one to the green light, and one to the blue light. By emitting a controlled combination of these three basic colours (red, green and blue), and hence stimulate the

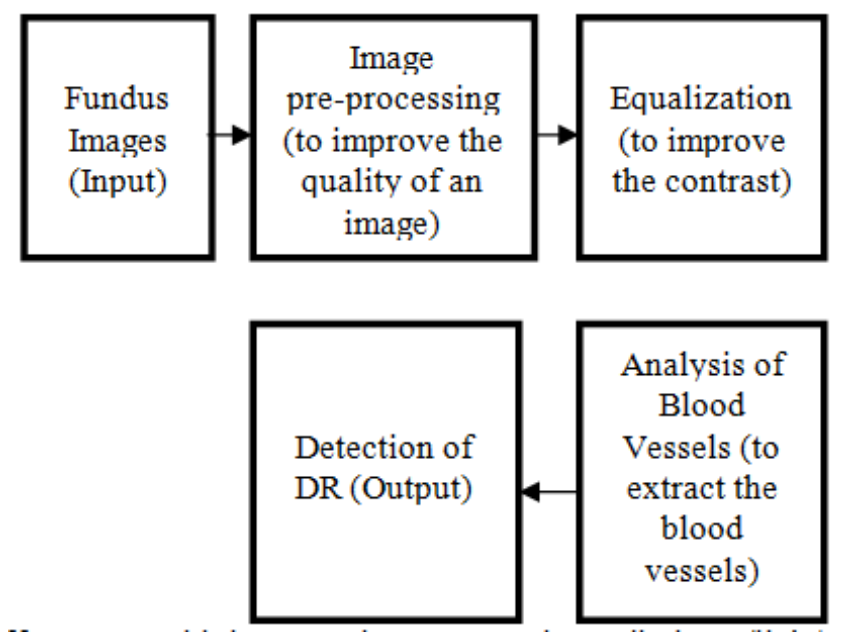

Figure No. 1. Block diagram of DR Detection using Image Processing Technique

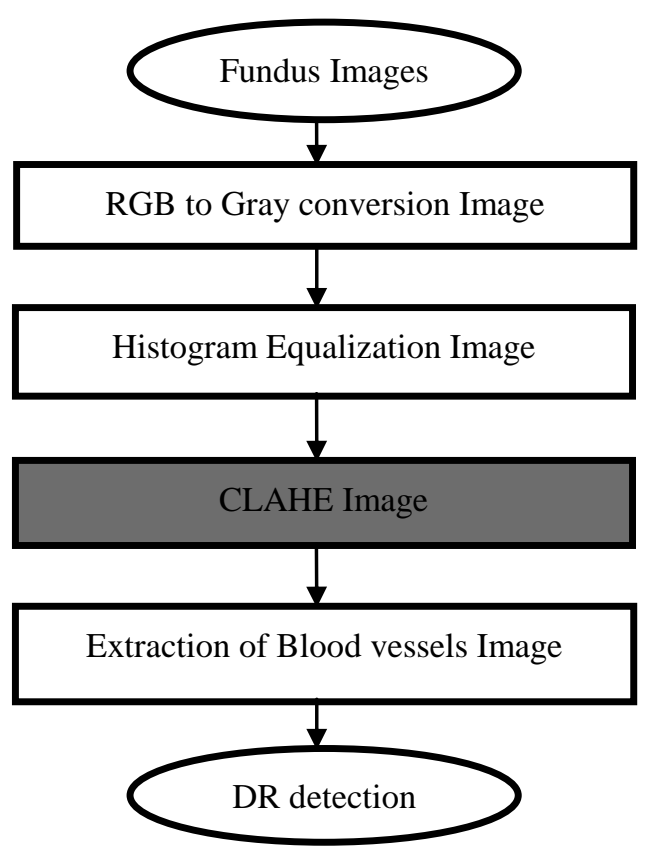

Figure No. 2. Flow chart of DR Detection using Image Processing Technique

The Retinal digital images are usually known as fundus image taken by digital fundus camera. The fundus image is the portion of the inner eye that can be seen during an eye examination by looking through the pupil. "Fundus" is the Latin word for the bottom. In medical field, fundus image refers to the bottom (or) base of an organ and it is the interior surface of the eye opposite to the lens and it includes the retina, optic disc, macula etc and then the fundus image can be examined by the ophthalmoscopy and/or by the fundus image photography. The term fundus image may also be inclusive of Bruch's membrane and the choroid. The Humans perceive colour through wavelength-sensitive sensory cells called cones. There are three different types of cones, each are with a different sensitivity to electromagnetic radiation (light) three types of cones which will be able to generate almost any perceivable colour.

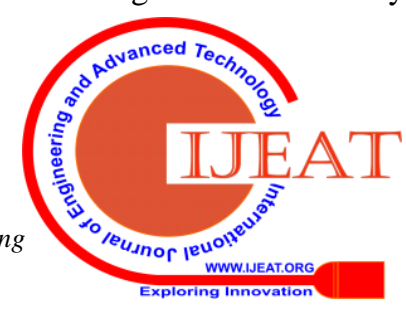


This is the reason that colour images are often stored as three separate image matrices. The one is storing the amount of red (R) in each pixel, one is the amount of green $(G)$ and one is the amount of blue (B). Such colour images are stored in an RGB format. In grayscale images, however, it cannot be differentiated how much emit of the different colours, but it can emit the same amount in each channel. It can differentiated as the total amount of emitted light for each pixel, little light gives dark pixels and much light is perceived as bright pixels. In image processing, the histogram equalization is a method by which it usually increases the global contrast for many images, especially when the usable data of the image is represented by close contrast values. By this adjustment of the intensities it can be better distributed on the histogram and this allows for the areas of lower local contrast to gain a higher contrast and the Histogram equalization accomplishes this by effectively spreading out the frequent intensity values.

The extraction of blood vessels includes the following processes. Many retinal diseases are characterized by changes in retinal vessels and the retinal vascular structure consists of two kinds of vessels: arteries and veins. An important symptom for Diabetic Retinopathy DR is irregularly wide veins, leading to an unusually low ratio of the average diameter of arteries to veins. After the extraction of blood vessels, DR can be detected and then for the final evaluation of accuracy of the proposed algorithm the following parameters are used,

1. Sensitivity (SE)

2. Specificity (SP)

3. Accuracy (ACC)

The equations for finding the above parameters are as follows. For Sensitivity it is given as, $\mathrm{SE}=\mathrm{TP} /(\mathrm{TP}+\mathrm{FN})$; For Specificity it is given as, $\mathrm{SP}=\mathrm{TN} /(\mathrm{TN}+\mathrm{FP})$; For Accuracy it is given as, $\mathrm{ACC}=(\mathrm{TP}+\mathrm{TN}) /(\mathrm{TP}+\mathrm{FN}+\mathrm{FP}+$ TN).

In TP and TN, T stands for correct detection.

In FP and FN, F stands for wrong detection.

Where, TP is True Positive

$\mathrm{TN}$ is True Negative

FP is False Positive

FN is False Negative

The percentage values of accuracy in the DR Detection using Image Processing are given in the Table I

Table I. Testing table for finding the Accuracy

\begin{tabular}{|c|c|c|c|}
\hline \multirow{2}{*}{$\begin{array}{c}\text { HRF } \\
\text { DATABASE }\end{array}$} & SENSITIVITY (SE) \% & SPECIFICITY (SP) \% & ACCURACY (ACC) \% \\
\cline { 2 - 4 } & 79.11 & 98.60 & 97.96 \\
\hline 02_h.jpg & 81.02 & 98.24 & 97.61 \\
\hline 04_h.jpg & 75.53 & 97.16 & 96.52 \\
\hline 06_h.jpg & 73.36 & 97.24 & 96.52 \\
\hline 08_h.jpg & 82.10 & 98.70 & 97.85 \\
\hline 10_h.jpg & 82.18 & 98.74 & 97.71 \\
\hline 12_h.jpg & 84.14 & 99.18 & 98.25 \\
\hline 03_dr.jpg & 83.46 & 99.15 & 98.14 \\
\hline 05_dr.jpg & 86.18 & 98.53 & 97.91 \\
\hline 07_dr.jpg & 86.22 & 98.61 & 97.93 \\
\hline 09_dr.jpg & & & \\
\hline
\end{tabular}

\section{RESULTS AND DISCUSSION}

The DR (Diabetic Retinopathy) Detection using Image Processing technique proposed in this paper is implemented by the software tool, MATLAB (R2010a) in which C programming language is used in it, are running on a $\mathrm{PC}$. The simulation results are obtained by the DR Detection using Image Processing Technique.

This method involves the processes in which affected DR is detected in the fundus image and the DR is not detected in the healthy fundus image.

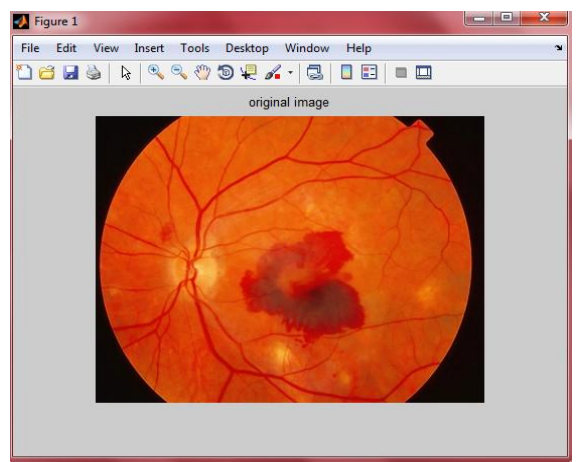

Figure No. 3. Original Image 
Diabetic Retinopathy Detection using Image Processing

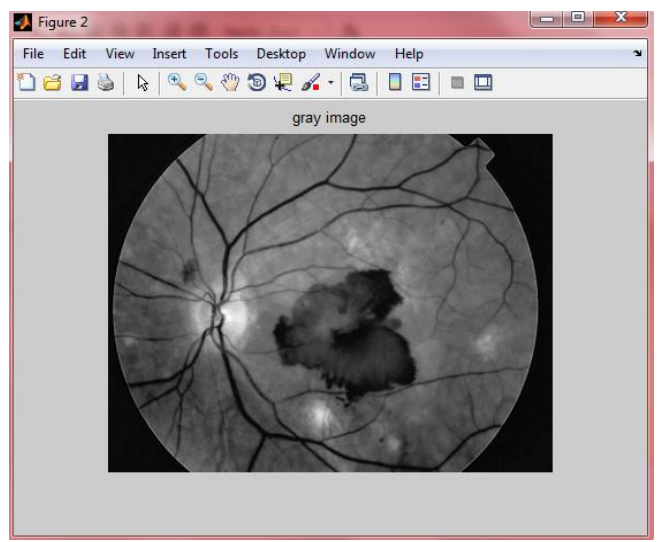

Figure No. 4. Gray Image

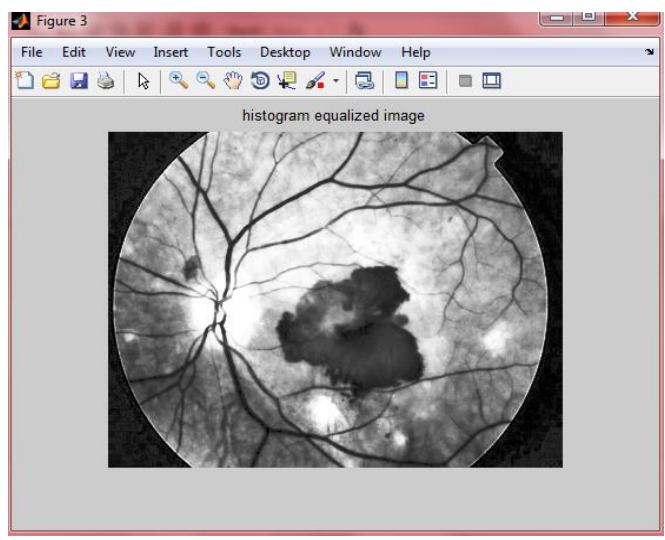

Figure No. 5. Histogram equalized Image

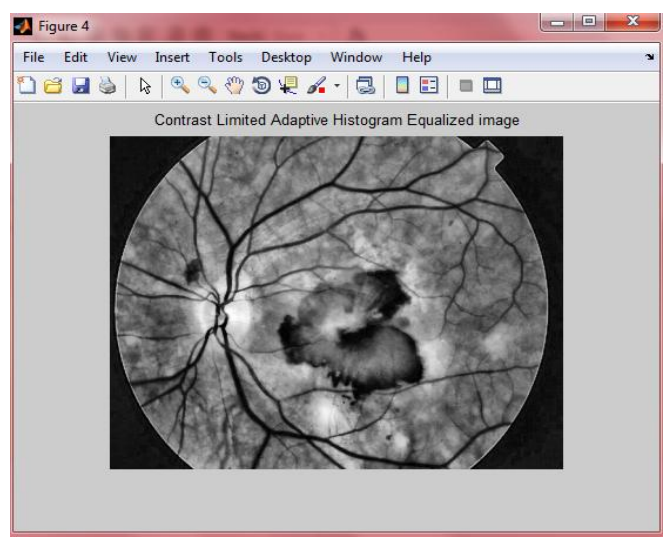

Figure No. 6. CLAHE Image

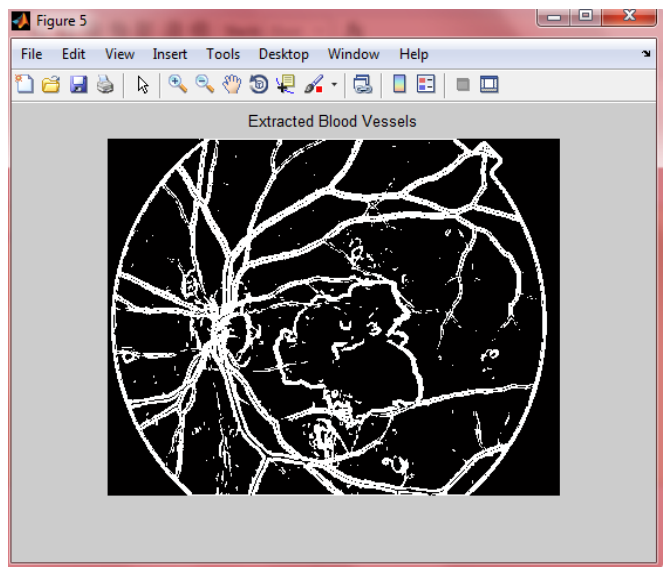

Figure No.7. Extracted Blood Vessels

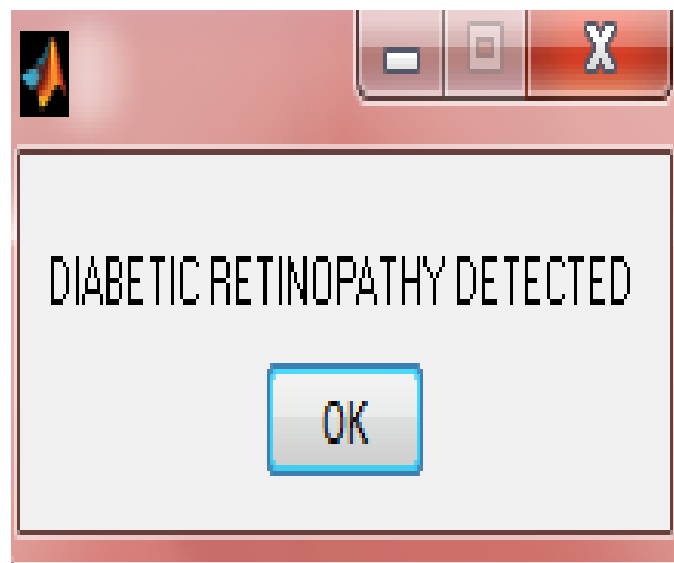

Figure No. 8. DR Detected

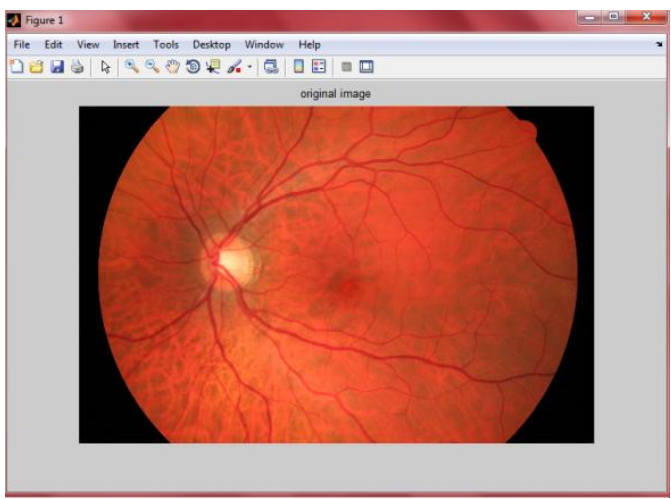

Figure No. 9. Original Image

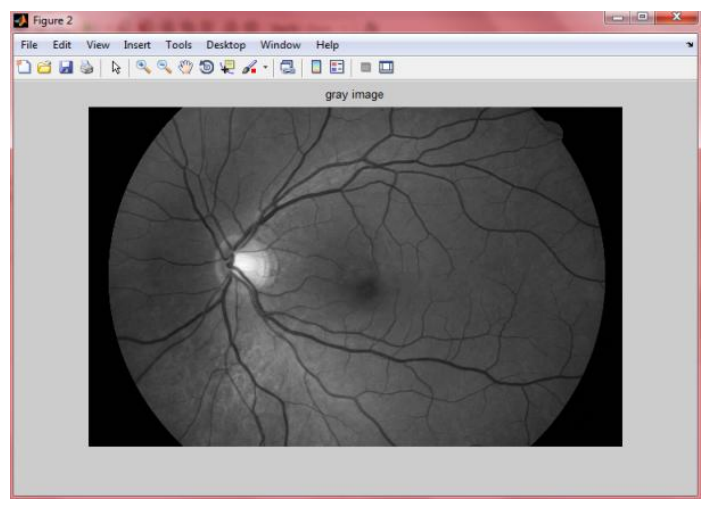

Figure No. 10. Gray Image

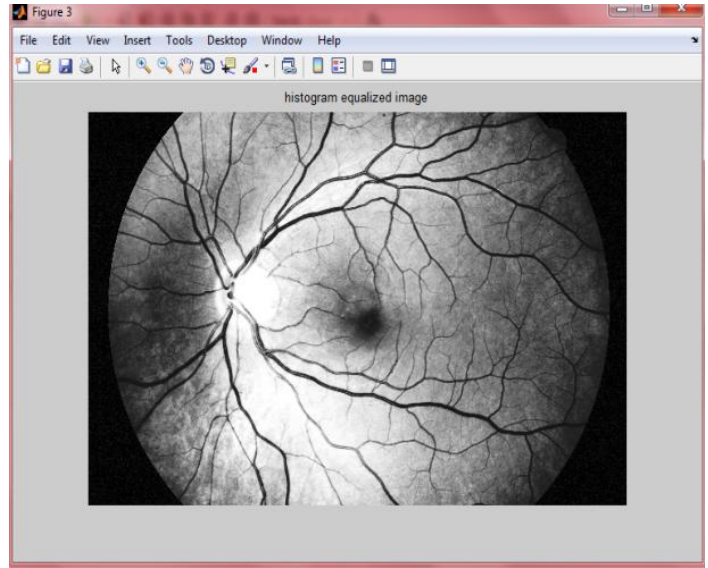

Figure No. 11. Histogram equalized Image

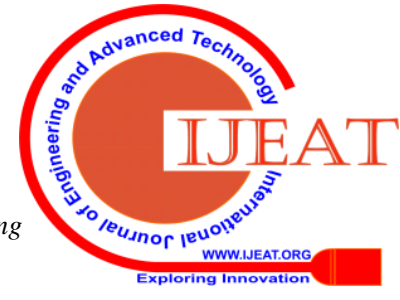




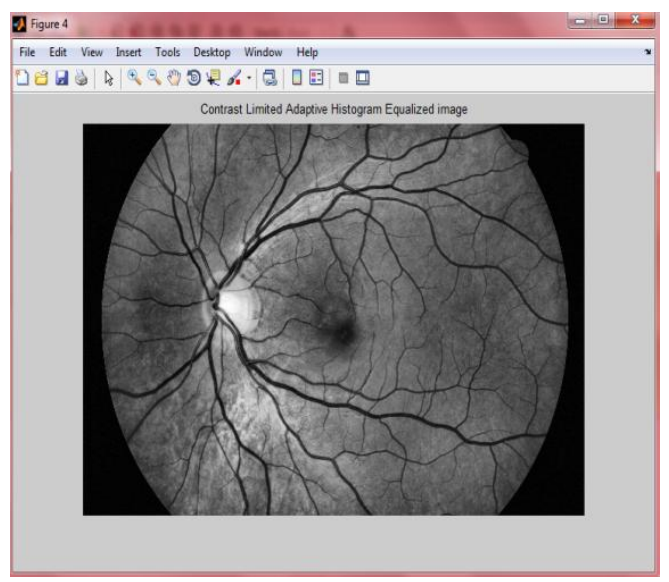

Figure No. 12. CLAHE Image

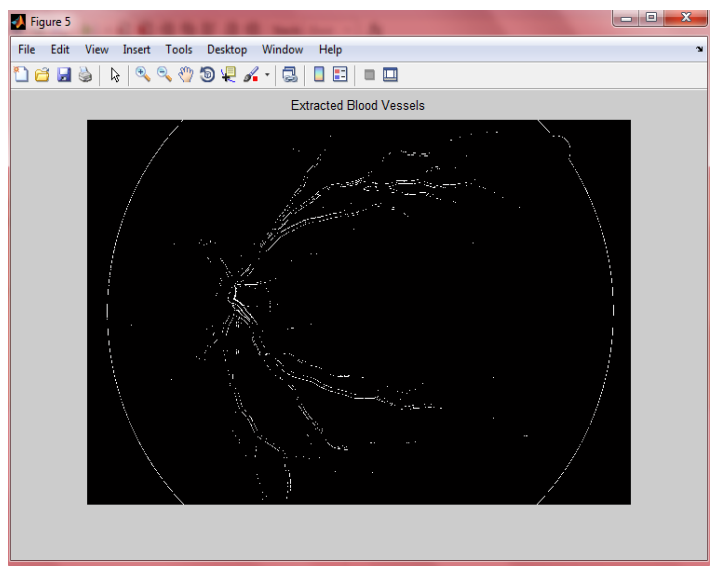

Figure No. 13. Extracted Blood Vessels

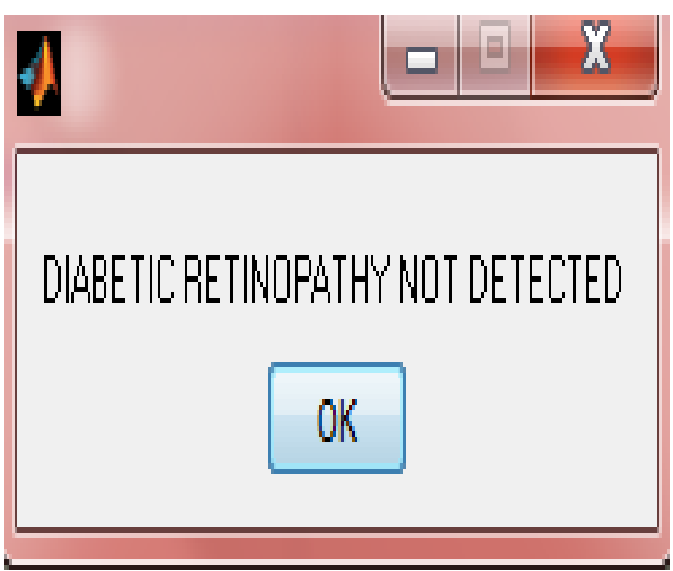

Figure No. 14. DR Not Detected

\section{CONCLUSION}

The result showed that from the affected fundus image the DR (Diabetic Retinopathy) disease is detected and then for the healthy fundus image it is found that DR is not affected. The final evaluation of Accuracy of the proposed algorithm is calculated by using Sensitivity (SE), Specificity (SP) and Accuracy (ACC) parameters. These parameters are calculated from the training images provided by the High Resolution Fundus (HRF) images database for DR detection. The training images are manually detected fundus images by ophthalmologists. Finally $98 \%$ of Accuracy is achieved in the detection of DR. Thus this project presents the work on the use of Image Processing Techniques for DR Detection.

\section{ACKNOWLEDGMENT}

Our sincere thanks to the Management of K.V.Subba Reddy College of Engineering for Women, Kurnool, AP and Sri Krishna College of Engineering and Technology, Coimbatore, Tamilnadu for rendering support for our work.

\section{REFERENCES}

1. Bansal, A., Vats, A., Jain, A., Dutta, M. K., Burget, R., \& Prinosil, J. (2016, June). An efficient automatic intensity based method for detection of macula in retinal images. In Telecommunications and Signal Processing (TSP), 2016 39th International Conference on (pp. 507-510). IEEE.

2. Elbalaoui, A., Fakir, M., Taifi, K., \& Merbouha, A. (2016, March). Automatic Detection of Blood Vessel in Retinal Images. In Computer Graphics, Imaging and Visualization (CGiV), 2016 13th International conference on (pp. 324-332). IEEE.

3. Ganguly, S., Ganguly, S., Srivastava, K., Dutta, M. K., Parthasarathi, M., Burget, R., \& Riha, K. (2014, November). An adaptive threshold based algorithm for detection of red lesions of diabetic retinopathy in a fundus image. In Medical Imaging, m-Health and Emerging Communication Systems (MedCom), 2014 International Conference on (pp. 91-94). IEEE.

4. Kumari, V. V., \& Suriyanarayanan, N. (2010). Blood vessel extraction using wiener filter and morphological operation. Int. J. Comput. Sci. Emerg. Technol, 1(4), 7-10.

5. Minar, J., Pinkava, M., Riha, K., Dutta, M. K., \& Sengar, N. (2015, October). Automatic extraction of blood vessels and veins using laplace operator in fundus image. In Green Computing and Internet of Things (ICGCIoT), 2015 International Conference on (pp. 23-26). IEEE.

6. Minar, J., Riha, K., Krupka, A., \& Tong, H. (2014). Automatic detection of the macula in retinal fundus images using multilevel thresholding. International Journal of Advances in Telecommunications, Electrotechnics, Signals and Systems, 3(1), 1316.

7. Thanapong, C., Watcharachai, W., \& Somporn, R. (2007, August). Extraction blood vessels from retinal fundus image based on fuzzy Cmedian clustering algorithm. In Fuzzy Systems and Knowledge Discovery, 2007. FSKD 2007. Fourth International Conference on (Vol. 2, pp. 144-148). IEEE.

8. Zaman, A. U., \& Bashir, S. K. (2016). Diabetic retinopathy detection using image-processing (Doctoral dissertation, BRAC University) 